\title{
Delayed Massive Traumatic Hematoma in the Corpus Callosum: Two Case Reports with Literature Review
}

\author{
Yanli Du, ${ }^{1}$ Zongli Han, ${ }^{2}$ Siyang Zheng, ${ }^{2}$ Tao $\mathrm{Wu}^{2}$ and Wei Yin ${ }^{2}$
}

A delayed massive traumatic hematoma in the corpus callosum is extremely rare. We report two cases with a delayed massive callosal hematoma caused by blunt head trauma. A massive callosal hematoma was diagnosed by computed tomography (CT) 2 weeks after a minor head injury in a 29-year-old man. A similar but larger hematoma developed 12 hours post-trauma with acute onset of consciousness disturbance in a 39-yearold man. Emergency CT angiography revealed no vascular pathologies in either case. The first patient was managed conservatively and recovered, whereas the second patient was treated surgically and died. The literature was reviewed regarding the possible mechanism of production of these lesions following head injury and therapeutic considerations are discussed.

Keywords: delayed hematoma, head trauma, corpus callosum, diffuse injury

\section{Introduction}

Since computed tomographic (CT) scans began to be used in the clinical field, deep-seated hemorrhagic lesions caused by head injuries have been clearly demonstrated. However, massive traumatic hematomas in the region of the corpus callosum (CC) have been found to be uncommon, and delayed hematoma in this region is very rare, although small hemorrhagic foci in the $\mathrm{CC}$ or surrounding structures are frequently seen in shearing injuries of the cerebral white matter ${ }^{1)}$ or in diffuse axonal injuries (DAIs). ${ }^{2,3)}$ Little is known about delayed hematomas in the $\mathrm{CC}$, and generally they are mentioned only incidentally among other findings. In this report, two cases with delayed massive hematoma of the $\mathrm{CC}$ produced by blunt head injury are described, and their possible dynamic mechanisms as well as clinical considerations are discussed.

\section{Case Reports}

\section{Case 1}

A 29-year-old man was admitted with complaints of gradually deteriorating dizziness for over 2 days and subsequent vomiting. His history revealed that he had been hit on the left occiput of his head by a golf ball 2 weeks previously, which

${ }^{1}$ School of Medical Technology and Nursing, Shenzhen Polytechnic, Shenzhen, P.R. China

${ }^{2}$ Department of Neurosurgery, Peking University Shenzhen Hospital, Shenzhen, P.R. China

Received: March 25, 2013; Accepted: January 21, 2014 had led him to be observed in our hospital because of headache. He was discharged with continuous headaches but without deterioration. The headache was deep-seated and relieved by the usual analgesics. The Glasgow Coma Scale (GCS) score was reported to be 15 on the initial neurological exam right after the trauma, and CT scan revealed no hematomas except some hemorrhage of the interhemispheric fissure cistern (Fig. 1A).

On his second admission, the patient was lethargic, had a stiff neck and slight paralysis of the right lower limb, and urinary incontinence, and the cranial CT disclosed a massive hyperdense mass in the $\mathrm{CC}$ from the genu to the splenium (Fig. 1B). Assuming that a ruptured traumatic aneurysm or an arteriovenous malformation caused the hematoma, an emergency computed tomography angiography (CTA) was performed, but it yielded no pathological results. Repeat CTA, digital subtraction angiography (DSA), and magnetic resonance imaging (MRI) were performed on days 4,7 , and 8 after the second admission, respectively, to explore the potential etiology of the hematoma. However, no pathology was noted (Fig. 2A-D). Blood count, blood chemistry, bleeding, and coagulation times were found to be normal. The patient had no systemic disease such as high blood pressure, diabetes, or liver disease, or was not using of aspirin or anticoagulant medications.

Because no neurological deterioration was observed after admission, conservative medical management was undertaken, including antiedematous treatment, antiepileptic therapy, anti-vasospasm therapy, and haemostatic therapy. The patient's condition improved gradually. Three weeks later, the patient was discharged with no obvious neurological deficit except slight left-handed apraxia. Three months later, a follow-up DSA image showed no demonstrable vascular abnormality (Fig. 2B).

\section{Case 2}

A 39-year-old man who had no demonstrable predisposing abnormalities was admitted approximately 2 hours after being involved in an accidental fall. He fell down from a height of about 3 metres, and he recovered after a transient period of unconsciousness. An emergency CT scan demonstrated subarachnoid hemorrhage (hemorrhage of the interhemispheric and right sylvian fissures) and right temporal and occipital bone fractures but no evidence of an intracranial hematoma (Fig. 1C). Coagulation function was normal.

Approximately 12 hours after the injury, the patient looked 


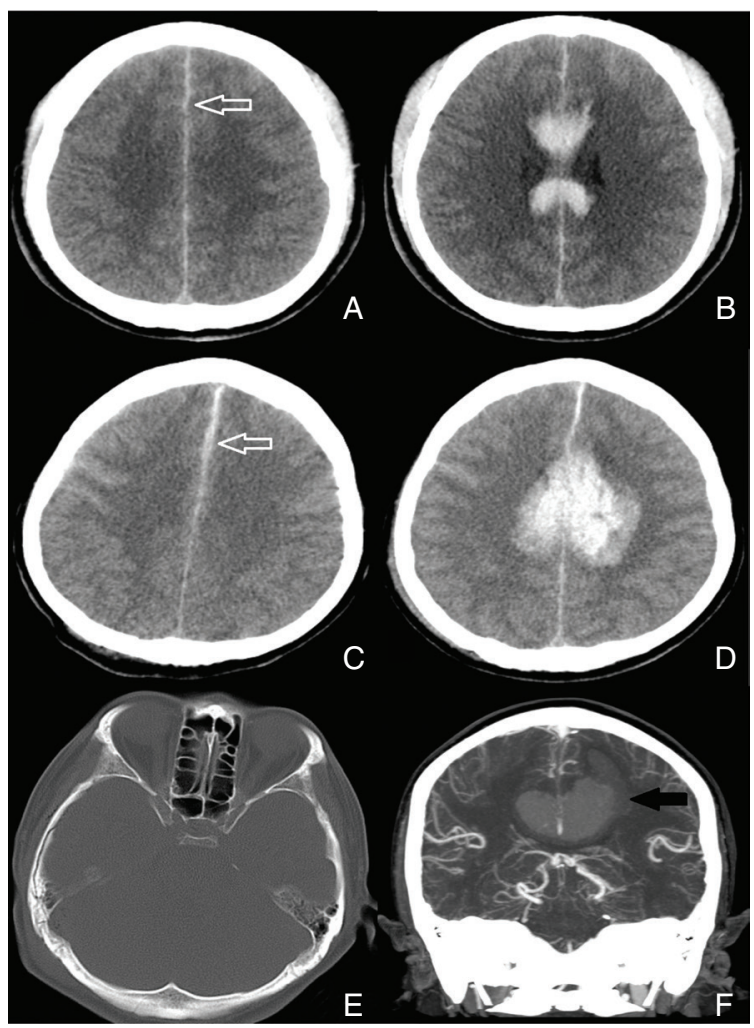

Fig. 1 Computed tomography (CT) information of the two cases (case 1: A and B; case 2: C-F). Onset of a massive hematoma of the corpus callosum on 2 weeks and 12-hours control-CT (B and D) not present at admission but with evident subarachnoid hemorrhage at the interhemispheric fissure (see white arrow in A and C). CT scan of the head showing no blood in the lateral ventricle. The line of fracture is oblique from the right occipital to the temporal bone and reaches the skull base (E). The avascular mass is mainly centrally located and extend from the genu to the splenium with slight deviation to the left (F see black arrow).

pale and he began yelling and then experienced acute onset of consciousness disturbance, right limb movement disorder, vomiting, and urinary incontinence. Physical examination disclosed a light coma with a GCS score of 9 (E2, V2, M5), brisk and equal corneal and light reflexes, and slight paralysis of the right lower limb.

A CT scan of the brain was obtained which revealed a massive hemorrhage of the $\mathrm{CC}$ extending from the genu to the body and the splenium without intraventricular hemorrhage (Fig. 1D). An emergency CTA was conducted to rule out the possibility of incidental rupture of an arteriovenous malformation or of aneurysms (Fig. 2E, F). Because of the neurological deterioration, surgery was undertaken without any further diagnostic measures. Severe swelling of the brain was discovered during surgery, so decompressive craniectomy and external ventricular drainage were performed. Postoperatively, the patient remained comatose with sensitive pupillary light reflex. Throughout the next 13 hospital days, bone window tension or intracranial pressure (ICP) were almost normal. The patient's condition deteriorated without evidence of

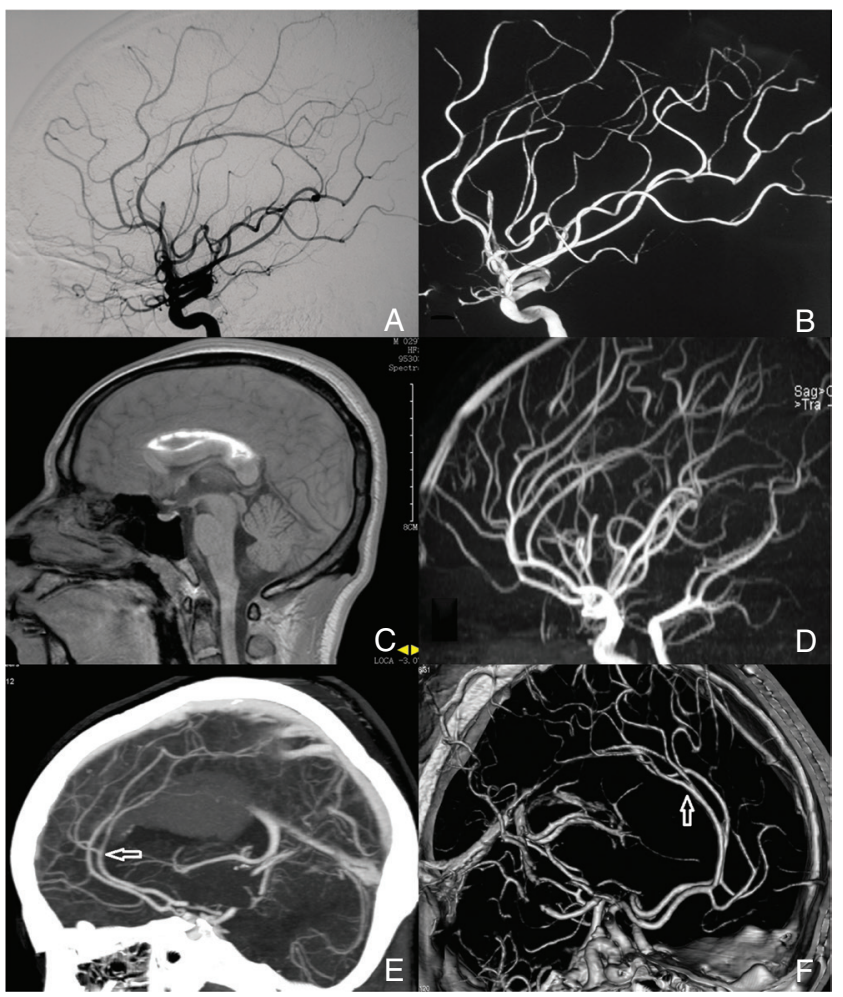

Fig. 2 Angiography information of the two cases (case 1: A-D; case 2: E and F). DSA and repeat DSA after second admission (at 7 days and 90 days, respectively). No aneurysm or malformation was detected (A and B, respectively). Magnetic resonance imaging $\left(\mathrm{T}_{1}\right)$ and angiography of the head showed the subacute hematoma extending from the genu to the splenium, but no demonstrable occupying lesion or vascular abnormality ( $\mathrm{C}$ and $\mathrm{D}$, respectively). Computed tomography angiography of the head showed no vascular lesions along the distal anterior cerebral artery (D and E), especially in the junction of the pericallosal and callosomarginal artery (see white arrows in $\mathrm{E}$ and $\mathrm{F}$ ). DSA: digital subtraction angiography.

further rebleeding on CT, and angiography was not performed due to the patient's poor medical condition. The patient died from pneumonia on the fourteenth day post-trauma. An autopsy was not performed because of local customs.

\section{Discussion}

The mechanisms that underlie traumatic injuries of the $\mathrm{CC}$ are poorly understood, and those that have been proposed are based mostly on autopsy findings. Some researchers reported that massive hematomas were produced by severe head injury and that the tentorial edge played a significant role in these lesions. ${ }^{4,5)}$ They found that the impact was situated at the vertex above the level of the $\mathrm{CC}$, and that the force of impact was directed toward the skull base. Another researcher suggested that the brain might be displaced at the moment of the impact in such a way that the $\mathrm{CC}$ strikes against the edge of the falx; he assumed that the sharp, free edge of the falx and tentorium made the CC more vulnerable. ${ }^{6}$ However, the falx is sufficiently close to the $\mathrm{CC}$ to allow damage by direct contact 
only in its caudal-most portions. Thus, the direct contact damage theory is not well applicable to lesions involving the entire CC, as was true for our two cases.

Much progress has been made in the development of sophisticated mathematical models (finite element model) of the brain's response to impact. These models provide stress and strain values for the various regions of the brain under various impact conditions. Nishimoto and Murakami ${ }^{7}$ found that with the falx cerebri removed, the shear stress concentration on the $\mathrm{CC}$ was reduced to $50 \%$ of that in the human model, demonstrating the effect of the falx cerebri structure on the high shear stress at the CC. Zhang et al. ${ }^{8,9)}$ found that lateral impact induced higher localized shear stress (up to 10 times) in the core regions of the brain and was more injurious than that predicted for frontal impact, ${ }^{4,5)}$ and Gennarelli et al. ${ }^{10)}$ found the lateral head acceleration also correlated highly with the production of focal lesion in the CC. In case 1, the hit was on the left occiput of the head directing toward the skull base by a stray golf ball without skull fracture which was also reported in the literature. ${ }^{11,12)}$ It is likely that angular acceleration and head rotation from the transfer of golf ball kinetic energy produce shearing forces, leading to parenchymal and underlying axonal (white matter) damage. In case 2, presented herein, the forces were assumed to be directed obliquely from the right temporo-occipital to the left frontotemporal areas of the brain; thus, it is likely that shear stress peaks initially appeared beneath the cortical surface, and then evolved toward the core regions of the brain ${ }^{9,13)}$ (Figs. 1E and 2C). The patient's (case 2) initial local neurological symptom was right hemiplegia, which suggests that the initial hemorrhagic spot was located to the left of the falx cerebri (also can be seen on the CT scan, Fig. 1F). This is consistent with the premise that the falx cerebri plays an important role in the mechanism. .) Clinically, the vast majority of callosal lesions are found in the body and splenium, ${ }^{14)}$ which is consistent with the fact that the thick fibers in the posterior midbody and splenium are more sensitive to rapid head acceleration and secondary injury changes following a traumatic event compared to the thin fibers. ${ }^{15)}$ Another reason is thought to be related to the shear strains that occur at the junction of the $\mathrm{CC}$ and the septum pellucidum and fornix when the cerebral hemispheres are markedly displaced. The rigid falx, which is broader posteriorly, prevents the cerebral hemispheres from moving across the midline, which further strains the connecting CC. Anteriorly the falx is shorter and allows transient displacement across the midline. ${ }^{2)}$ However, when the shear and strain loading of the CC is large enough, the anterior portion of the CC will also be affected. This could explain the involvement of the entire $\mathrm{CC}$ during the injury.

Delayed post-traumatic intracranial hematoma was first described by Bollinger as Spät-Apoplexie ${ }^{16)}$ because patients developed an apoplectic onset of intracerebral hematoma weeks or months after a head injury. The mechanism responsible for it is still controversial. Although some researchers reported that disseminated intravascular clotting and fibrinolysis were responsible for the development of $90 \%$ of delayed traumatic intracranial hemorrhage, ${ }^{17)}$ our two cases showed no evidence of coagulation dysfunction. The hematomas in the $\mathrm{CC}$ were not secondary to hemorrhagic infarction, as the CT scans demonstrated no low density areas in the CC and no angiographic evidence of narrowing or obstruction of the anterior cerebral arteries (Fig. 2). Primary direct contusion likely was a major factor in the evolution of the hematoma in the $\mathrm{CC}$, as penetrating cortical medullary arteries in the CC have their major sweep in the coronal plane and may be vulnerable to shear and stress due to impact along the long axis of the skull. ${ }^{18)}$ In addition, hemorrhage of the interhemispheric fissure cistern along with the hematoma was found in both of our cases, and this was also found in other studies, 3,19 ) this may be associated with the delayed hematoma of the CC. Although traumatic intracranial aneurysm is one of the causes of delayed apoplexy in patients with severe head trauma ${ }^{20)}$ following direct mural injury or accelerationinduced shearing force,$^{21)}$ neither of our cases showed this vascular abnormality through CTA and/or DSA. However, we could not entirely eliminate the possibility of traumatic aneurysm bleeding because the diagnostic accuracy of CTA may not allow detection of abnormalities of peripheral cerebral arteries (like our case 2) and spontaneous thrombosis of small intracranial aneurysms. ${ }^{22)}$ We could not pinpoint one particular factor responsible for the delayed hematoma. However, continuous headaches after discharge was observed in case 1 , and this symptom may have been indicative of subtle intracranial hemorrhage and subsequently with progressive dizziness. This pattern illustrates the chronic course of this patient's condition versus the acute onset typical of aneurysm rupture, which usually is combined with intraventricular hemorrhage or frontal hematoma. ${ }^{2}$ We hypothesize that the patient had some degree of asymptomatic derangement of the microvasculature of the $\mathrm{CC}$, that made the microvasculature more vulnerable to stretching and/or shearing forces; this condition predisposed the patient to hematoma formation when subjected to trauma. ${ }^{23)} \mathrm{We}$ assumed that the initial hemorrhage spot on the CC caused by the mild impact was minimal enough that it was either undetectable even with high-definition CT or that it had been already washed out when the first CT was performed; thus no obvious local neurological sign was apparent right after the injury, but it gave rise to symptoms several weeks later, presenting as a massive hematoma. ${ }^{24,25)}$ Additionally, posttraumatic headache may mask underlying chronic bleeding which also prolonged the course. Detailed cadaveric analysis of the microvasculature in patients who succumb to delayed traumatic hematoma of the $\mathrm{CC}$ may be helpful to determine the underlying etiology.

Adams et al. ${ }^{26)}$ reported that focal injuries of the $\mathrm{CC}$ and of the dorsolateral rostral brain stem near the superior cerebellar peduncle and pathological evidence of axonal disruption are characteristically seen in DAIs with progressive worsening of outcome. However, other researchers stated that these pathological "marker lesions" of DAI are commonly found not only in patients with diffuse brain injury but also in those with focal brain injury, ${ }^{2,27)}$ and they 
suggested that the term "DAI" should be used to represent a spectrum of injuries rather than a distinct well-defined clinical entity. ${ }^{28)}$

Our two cases exhibited some of the characteristics of DAI, but the lucid interval evident in our patients is usually absent in severe DAI, ${ }^{29,30)}$ and callosal hemorrhage was massive in our cases. Therefore, the stretching and/or shearing of the $\mathrm{CC}$ at the point of impact could have been more severe and extensive in our cases than in those described previously. ${ }^{26,27,31)}$ In case 1 , the patient was asymptomatic for 2 weeks because the trauma was not severe enough to alter the GCS score, and secondary factors (e.g., hypoxia, hypercarbia, hypertension, vasoparalysis, bleeding diathesis, and alcohol intake) $)^{24)}$ were absent.

Clinically, the neurological status of patients with traumatic callosal injury is usually grave and the mortality rate is high. ${ }^{4,19,32)}$ Poor outcome is associated with increasing age, low admission GCS, and the presence of space-occupying lesions, ${ }^{33,34)}$ especially those that involve both the genu and the splenium. ${ }^{3)}$ In our case 2, the patient's GCS level was 15 upon admission, and he experienced immediate onset of coma and underwent emergency surgery. In case 2, a relatively low ICP was observed postoperatively until the patient died. The association with DAI may explain why hematoma evacuation rarely benefits the patient, as it may induce additional damage to the severely injured brain. In our opinion, conservative treatment may be the optimal choice if the patient's neurological status is stable (as in case 1); otherwise, early ventricular drainage, plus decompressive craniectomy is an option if the ICP is high although the prognosis is poor.

\section{Conclusion}

Two patients with massive traumatic hematoma of the CC that was not immediately apparent upon presentation are described. Case 1 exhibited steady neurological status, was treated conservatively, and was discharged with no obvious neurological deficit. However, case 2 became comatose and died postoperatively. Potential etiology should be sought in patients with vascular disease, especially in cases with hemorrhage of the interhemispheric fissure cistern. Management is controversial, with surgery mandatory only for cases of progressive neurologic deficit. This information presented herein is likely to be helpful to surgeons faced with the practical problem of making management decisions about patients with this rare condition.

\section{Acknowledgment}

The authors thank Mr. Hui Qi who provided the DSA imaging and helped us to interpret the CTA and DSA imaging data.

\section{Conflicts of Interest Disclosure}

The authors declare that they have no conflicts of interests.

\section{References}

1) Zimmerman RA, Bilaniuk LT, Genneralli T: Computed tomography of shearing injuries of the cerebral white matter. Radiology 127:
393-396, 1978

2) Gentry LR, Thompson B, Godersky JC: Trauma to the corpus callosum: MR features. AJNR Am J Neuroradiol 9: 1129-1138, 1988

3) Matsukawa H, Shinoda M, Fujii M, Takahashi O, Yamamoto D, Murakata A, Ishikawa R: Genu of corpus callosum as a prognostic factor in diffuse axonal injury. J Neurosurg 115: 1019-1024, 2011

4) Komatsu S, Sato T, Kagawa S, Mori T, Namiki T: Traumatic lesions of the corpus callosum. Neurosurgery 5: 32-35, 1979

5) Lindenberg R: Significance of the tentorium in head injuries from blunt forces. Clin Neurosurg 12: 129-142, 1964

6) Peerless SJ, Rewcastle NB: Shear injuries of the brain. Can Med Assoc J 96: 577-582, 1967

7) Nishimoto T, Murakami S: Relation between diffuse axonal injury and internal head structures on blunt impact. J Biomech Eng 120: 140-147, 1998

8) Zhang L, Yang KH, King AI: Comparison of brain responses between frontal and lateral impacts by finite element modeling. $J$ Neurotrauma 18: 21-30, 2001

9) Zhang L, Yang KH, King AI: A proposed injury threshold for mild traumatic brain injury. J Biomech Eng 126: 226-236, 2004

10) Gennarelli TA, Thibault LE, Adams JH, Graham DI, Thompson CJ, Marcincin RP: Diffuse axonal injury and traumatic coma in the primate. Ann Neurol 12: 564-574, 1982

11) Etgen $T$, Sander K: Intracerebral haematoma without skull fracture by golf ball. BMJ Case Rep 2008: bcr0620080024, 2008

12) Nguyen J, Kaplan AM: Golf ball-related head injuries in children. Pediatr Neurol 39: 126-128, 2008

13) Viano DC, Casson IR, Pellman EJ, Zhang L, King AI, Yang KH: Concussion in professional football: brain responses by finite element analysis: part 9. Neurosurgery 57: 891-916; discussion 891-916, 2005

14) Gentry LR, Godersky JC, Thompson B: MR imaging of head trauma: review of the distribution and radiopathologic features of traumatic lesions. AJR Am J Roentgenol 150: 663-672, 1988

15) Kallakuri S, Li Y, Zhou R, Bandaru S, Zakaria N, Zhang L, Cavanaugh JM: Impaired axoplasmic transport is the dominant injury induced by an impact acceleration injury device: an analysis of traumatic axonal injury in pyramidal tract and corpus callosum of rats. Brain Res 1452: 29-38, 2012

16) Morin MA, Pitts FW: Delayed apoplexy following head injury ("traumatische spät-apoplexis"). J Neurosurg 33: 542-547, 1970

17) Kaufman HH, Moake JL, Olson JD, Miner ME, duCret RP, Pruessner JL, Gildenberg PL: Delayed and recurrent intracranial hematomas related to disseminated intravascular clotting and fibrinolysis in head injury. Neurosurgery 7: 445-449, 1980

18) Fujitsu K, Kuwabara T, Muramoto M, Hirata K, Mochimatsu Y: Traumatic intraventricular hemorrhage: report of twenty-six cases and consideration of the pathogenic mechanism. Neurosurgery 23: 423-430, 1988

19) Shigemori M, Kojyo N, Yuge T, Tokutomi T, Nakashima H, Kuramoto $S$ : Massive traumatic haematoma of the corpus callosum. Acta Neurochir (Wien) 81: 36-39, 1986

20) Komiyama M, Morikawa T, Nakajima H, Yasui T, Kan M: "Early" apoplexy due to traumatic intracranial aneurysm-case report. Neurol Med Chir (Tokyo) 41: 264-270, 2001

21) Nakstad P, Nornes H, Hauge HN: Traumatic aneurysms of the pericallosal arteries. Neuroradiology 28: 335-338, 1986

22) Vulekovic P, Karan M, Kojadinovic Z, Papic V, Djilvesi D, Mitic G: Spontaneous thrombosis of basilar artery aneurysm after subarachnoidal hemorrhage in a patient with thrombophilia and recurrent deep venous thrombosis. Clin Appl Thromb Hemost 17: 348-351, 2011

23) Jain SK, Sundar IV, Sharma V, Prasanna KL, Kulwal G, Tiwari RN: Bilateral large traumatic basal ganglia haemorrhage in a conscious adult: a rare case report. Brain Inj 27: 500-503, 2013

24) Fukamachi A, Nagaseki Y, Kohno K, Wakao T: The incidence and developmental process of delayed traumatic intracerebral haematomas. Acta Neurochir (Wien) 74: 35-39, 1985

25) Nanassis K, Frowein RA, Karimi A, Thun F: Delayed post-traumatic intracerebral bleeding. Delayed post-traumatic apoplexy: "Spatapoplexie". Neurosurg Rev 1(12 Suppl): 243-251, 1989

26) Adams JH, Graham DI, Murray LS, Scott G: Diffuse axonal injury due to nonmissile head injury in humans: an analysis of 45 cases. 
Ann Neurol 12: 557-563, 1982

27) Shigemori M, Kikuchi N, Tokutomi T, Ochiai S, Kuramoto S Coexisting diffuse axonal injury (DAI) and outcome of severe head injury. Acta Neurochir Suppl (Wien) 55: 37-39, 1992

28) Wilberger JE, Rothfus WE, Tabas J, Goldberg AL, Deeb ZL: Acute tissue tear hemorrhages of the brain: computed tomography and clinicopathological correlations. Neurosurgery 27: 208-213, 1990

29) Parker JR, Parker JC, Overman JC: Intracranial diffuse axonal injury at autopsy. Ann Clin Lab Sci 20: 220-224, 1990

30) Slazinski T, Johnson MC: Severe diffuse axonal injury in adults and children. J Neurosci Nurs 26: 151-154, 1994

31) Godersky JC, Gentry LR, Tranel D, Dyste GN, Danks KR: Magnetic resonance imaging and neurobehavioural outcome in traumatic brain injury. Acta Neurochir Suppl (Wien) 51: 311-314, 1990

32) Lindenberg R, Fisher RS, Durlacher SH, Lovitt WV, Freytag E: Lesions of the corpus callosum following blunt mechanical trauma to the head. Am J Pathol 31: 297-317, 1955

33) Atzema C, Mower WR, Hoffman JR, Holmes JF, Killian AJ, Wolfson AB; National Emergency X-Radiography Utilization Study (NEXUS) II Group: Prevalence and prognosis of traumatic intraventricular hemorrhage in patients with blunt head trauma. J Trauma 60: 1010-1017; discussion 1017, 2006

34) Zheng WB, Liu GR, Kong KM, Wu RH: Coma duration prediction in diffuse axonal injury: analyses of apparent diffusion coefficient and clinical prognostic factors. Conf Proc IEEE Eng Med Biol Soc 1: $1052-1055,2006$

Corresponding author:

Zongli Han, MD, MS, Department of Neurosurgery, Peking University Shenzhen Hospital, 1120 Lianhua Road, Futian District, Shenzhen, Guangdong Province, Shenzhen 518036, China.

$\triangle$ hanz1007@163.com 